Para enlazar con este artículo / To link to this article:

http://dx.doi.org/10.14198/fem.2019.34.06

Para citar este artículo / To cite this article:

Leguen, Brigitte. «Autofiction versus écriture de soi chez les écrivaines françaises contemporaines». En Feminismo/s, 34 (diciembre 2019): 121-141. Dosier monográfico: Estado actual de la investigación en Literatura francesa y Género: balance y nuevas perspectivas / État présent de la recherche en Littérature française et Genre: bilan et nouvelles perspectives, coord. Ángeles Sirvent Ramos, DOI: 10.14198/fem.2019.34.06

\title{
AUTOFICTION VERSUS ÉCRITURE DE SOI CHEZ LES ÉCRIVAINES FRANÇAISES CONTEMPORAINES
}

\section{AUTOFICTION VERSUS WRITING THE SELF IN CONTEMPORARY FRENCH WOMEN WRITERS}

\author{
Brigitte LEGUEN \\ UNED, Madrid \\ bleguen@flog.uned.es \\ https://orcid.org/0000-0001-8767-5980
}

\section{Résumé}

Nous analyserons dans cet article l'évolution de la littérature d'autofiction produite par des écrivaines du champ littéraire français, en nous centrant plus spécifiquement sur la production du XXI ${ }^{\mathrm{e}}$ siècle. Nous mettrons l'accent sur un thème qui a donné lieu à un débat médiatique important, celui des récits sur la vie sexuelle ainsi que sur toute la problématique de l'identité telle qu'elle s'exprime dans l'écriture de soi.

Mots-clé: Ecriture femme; féminisme; autofiction; sexualité; identité.

\begin{abstract}
In this article we analyze the evolution of autofiction produced by French women writers focusing more specifically on the $21^{\text {th }}$ Century literature. We will pay particular attention to an issue that is especially relevant in today's media: Narration on sexual life and a discussion about identity as manifested in writing the self.
\end{abstract}

Keywords: Women and writing; Feminism; Autofiction; Sexuality; Identity.

Los contenidos de la revista se publican bajo una licencia de Creative Commons Reconocimiento 4.0 Internacional (CC BY 4.0)

Feminismo/s 34, diciembre 2019, pp. 121-141 
Parler de soi n'a rien de nouveau et à chaque époque nous constatons la présence d'écrivains et d'écrivaines qui parlent d'eux-mêmes et de leur vie intime. Mais le sort des femmes au XX ${ }^{\mathrm{e}}$ siècle et cet Autre que Simone de Beauvoir pointait du doigt traverse l'espace autofictif avec de nouvelles perspectives et de nouveaux modèles.

Le terme on le sait est un néologisme apparu en 1977 sous la plume de Serge Doubrovsky qui l'emploie sur la quatrième de couverture du livre intitulé Fils. Ce mot a connu depuis lors un écho toujours grandissant et il a été employé de très diverses manières. C'est ce qu'on pourrait définir comme un mot-valise qui fait appel à l'autobiographie et à la fiction littéraire. Pour faire court on dira que l'autofiction est un détournement fictif de l'autobiographie.

En fait, toute définition de l'autofiction passe par une critique de l'autobiographie et se construit par rapport à elle ou contre elle, d'où le titre de cet article. Avec la découverte de l'inconscient, le sujet prend acte de sa fictionnalité et remet en question la véracité imperturbable des souvenirs et de leur récit. A partir des années soixante-dix, l'écriture du moi se caractérise par un permanent questionnement sur les limites de sa validité et le métadiscours devient une partie intégrante du discours littéraire. On assiste alors à une démultiplication du récit qui problématise l'écriture du moi sans cependant s'en éloigner.

Bien que l'intention de cet article ne soit pas de faire un retour méthodique sur les aspects théoriques mais bien plutôt de s'intéresser à la diversité des œuvres représentatives de ce qu'aujourd'hui nous considérons comme de l'autofiction, et bien que dans certains cas les auteures elles-mêmes s'y résistent, nous tenterons de délimiter brièvement ses caractéristiques essentielles.

Lautofiction est singulière et rencontre dès son origine des détracteurs acharnés au niveau du débat théorique la concernant. Elle s'assortit de deux clauses fondamentales si l'on part de la définition inaugurale de l'incontournable Doubrovsky pour qui l'autofiction est une fiction d'évènements et de faits strictement réels. La première clause est nominale et référentielle; l'homonymat entre l'auteur, le personnage et le narrateur est requis dans tous les cas. Cette exigence différentie clairement l'autofiction du roman autobiographique dans lequel l'auteur crée son propre alter ego. La seconde clause est modale et stylistique et, toujours selon Doubrovsky, l'autofiction est la fictionnalisation du vécu et cette fiction se fabrique grâce à l'organisation narrative et au style.

Feminismo/s 34, diciembre 2019, pp. 121-141 
Autrement dit, l'auteur part du référentiel puis il s'en détourne porté par l'imagination et la mise en texte. Cette définition sera suivie de bien d'autres qui remettent en question la référence indubitable, la vérité littérale, la cohérence historique au profit d'une autre perception plus fragmentaire et même arbitraire, soumise aux sursauts de la mémoire, au rôle de l'inconscient et à une perception temporelle plus subtile comme l'a bien montré Paul Ricoeur.

L'écrivain, à la différence du chercheur, assume son référentiel et sa fabulation dans le processus de création. La définition la plus proche de cette attitude face à l'autofiction serait celle que propose Marie Darrieussecq qui la décrit comme un pacte contradictoire qui se donne à la fois comme référentiel (elle se soumet à un principe d'exactitude factuelle) et non référentiel puisqu'elle se présente comme un roman, attestant ainsi son entrée en fiction. «Lautofiction -dit-elle- demande à être crue, et demande à être non crue» (3). Cette ambiguité ou hybridité évoquée en son temps par Philippe Lejeune problématise évidemment les rapports de l'auteur à lui-même et au monde qui l'entoure.

Lautofiction s'affirme donc comme une exception contradictoire qui concilie deux vecteurs apparemment antagoniques -factuel et fictif-. Selon les auteurs et par tout un jeu très variable comme le montrent bien les écrivaines dont nous allons parler, le fictif y devient référentiel et le référentiel se fictionnalise (prenons-en à témoins des auteurs tels que Annie Ernaux ou Patrick Modiano).

D'autre part, et cela en marge de la qualité littéraire et esthétique du texte, l'autofiction est engagée souvent contre son gré dans un procès moral chargé d'accusations diverses comme l'impudeur, le narcissisme, l'amoralité qui concerne plus les effets médiatiques du procédé que ses conséquences littéraires.

Si on tient compte du fait que l'écriture sur soi est indissociable d'un dialogue avec la société (Bereni et Tranchman 17) et que les rapports de genres sont toujours impliqués dans d'autres rapports de pouvoir, on comprend mieux que l'autofiction au féminin ne puisse être lue sans tenir compte de cette perspective (sujet social/artiste). L'impératif phallologocentrique énoncé par Hélène Cixous continue d'être un élément dont il faut tenir compte dans l'analyse des contraintes sociales et symboliques qui pèsent sur le travail de l'artiste. 
Il existe beaucoup de détours dans la lutte des stéréotypes: choisir la création de personnages masculins, mettre au cœur de l'œuvre la question des identités sexuées, jouer sur l'absence de marque de genre, revisiter les genres littéraires et accorder une place nouvelle aux personnages féminins. Ce dernier aspect est celui que choisissent les écrivaines de l'autofiction. Il s'agira donc de se pencher sur les productions de l'espace fictif en tenant compte de ces conditions et en en mesurant les effets sur les textes évoqués.

Afin d'entreprendre ce bilan partiel de la littérature française contemporaine tenant compte de l'explosion des écritures de soi selon diverses modalités, nous adopterons le terme proposé par Havercroft -l'espace autofictif- et nous suivrons l'évolution récente de ce type de fiction dans la perspective des années 2000 (tout en faisant référence inévitablement à la littérature antérieure).

Il ne fait aucun doute que de Serge Doubrovsky à Christine Angot, de Fils publié en 1977 à Le Marché des amants publié en 2008, tout a bien changé, surtout si l'on se centre sur les textes produits par des femmes. On assiste au cours de ces dernières années à un grand «déballage» très proche de la littérature industrielle qui fait souvent l'éloge du non style. Certains thèmes prennent de l'ampleur et répondent au désir d'indigner et de provoquer comme on le verra plus avant. La sexualité sous tous ses jours, la physiologie de l'intimité à tous ses degrés, les misères du corps meurtri, malade, blessé, âgé sont parmi les thèmes majeurs de l'autofiction contemporaine qui déploie une panoplie souvent sordide propre d'un univers hardcore, d'une impudeur radicale et provocatrice souvent accompagnée d'un sulfureux parfum de scandale qui vend bien.

Cette tendance à l'abjection (qui n'est pas complètement nouvelle, souvenons-nous de l'exemple de Rachilde) répond probablement au désir de secouer un joug qui a trop longtemps pesé sur la «bienséance» qu'on attendait des discours au féminin et qui a donné lieu à une censure dont parfois on s'étonne dans nos sociétés occidentales apparemment émancipées (rappelons le cas sanglant de Violette Leduc).

La violence du langage, le concret et le factuel, l'antiroman sentimental si longtemps investi par les femmes va très certainement dans le sens du féminisme contemporain et coincide avec la volonté d'occuper un champ et de sortir des stéréotypes étouffants et persistants qui continuent à sévir dans

Feminismo/s 34, diciembre 2019, pp. 121-141 
la critique actuelle (nous y reviendrons avec Virginie Despentes et son essai King Kong Théorie).

Le grand risque d'un tel investissement du réel et du concret dans le récit serait bien sûr une réduction du genre autofictif en simple confession/ déballage refusant toute place à la littérature (la haute littérature face à la littérature de masse). Un monde séparerait-il les textes de Christine Angot, Catherine Cusset ou Nelly Arcan des textes aux exigences stylistiques et formelles d'Anne Garréta (Pas un jour, 2002) ou de Camille Laurens (Ni toi ni moi, 2006)?

Le «tout-dire» déjà revendiqué au $\mathrm{XX}^{\mathrm{e}}$ siècle par Leduc, Duras, Beauvoir, Ernaux, l'est aussi par les romancières les plus contemporaines. Accusé d'impudique, le roman d'autofiction serait surtout exhibitionniste de façon parfois infondée, bien que pas toujours: Les souvenirs d'enfance de Ernaux, Arcan, Delaume, Angot, sont suffisamment traumatisants pour expliquer le besoin de les mettre en récit. Toutes pourtant ne se rangent pas sous la bannière de l'autofiction, comme Annie Ernaux qui s'y refuse -bien qu'on l'y place- et qui revendique un rapport de soi avec la réalité sociohistorique produisant une ethno-socio-biographie qui va au-delà de l'anecdote personnelle et qui débouche sur une autobiographie impersonnelle (voir par exemple son texte Les Années, 2008); ce que Bruno Blanckman classe dans la catégorie des récits transpersonnels.

Il existe d'autre part, dans de nombreux cas, une étroite relation entre l'autofiction et un certain type d'engagement. Lautofiction, telle qu'elle est pratiquée par les écrivaines contemporaines, est le symptôme des transformations sociales, mentales, historiques qui caractérisent la postmodernité et plus particulièrement l'évolution des genres telle que les femmes la vivent actuellement. Elle révèle les traits récurrents de notre postmodernité, l'égotisme forcené, la présence obsédante du sexe et du corps, et d'autres aspects que Gilles Lipovetsky décrivait déjà fort bien dans son livre L'Ère du vide (dont la première édition est de 1983).

À la suite de cette perspective des années 80 , on constate une prolifération d'opinions toujours viables et se ralliant au même résultat: Le narcissisme et l'externalisation généralisée de Philippe Muray (2005), la fragmentation du moi de Christopher Lasch auteur de La Culture du Narcissisme (1979), la 
volonté d'«extimiser» le malaise, un autre terme très utilisé qui fait allusion, une fois de plus, au déballage de l'intimité.

Dans tous les cas, les différences seront soit d'ordre esthétique, soit d'ordre éthique. Certaines chercheront à produire une écriture sans style, littérale, d'autres transcenderont le moi en recherchant un style particulier.

Certaines caractéristiques s'appliquent à tous, eux et elles: la marchandisation de la culture selon la terminologie de Bourdieu, la présence toujours plus envahissante des médias et des réseaux sociaux dans une société du spectacle (Debord) qui revendique l'accès immédiat à tous les produits de consommation.

La pornographie ou l'érotisme en littérature écrite par des femmes n'est pas non plus une nouveauté: Histoire d'O de Pauline Réage (pseudonyme de Dominique Aury) ou le fameux Journal d'Anaïs Nin en sont la preuve brillante mais ce qui est nouveau c'est d'une part la massification et la vulgarisation de la production littéraire, et d'autre part, l'accès des femmes à une liberté d'expression et à une capacité d'expression à laquelle la société n'était pas habituée dans la sphère publique.

$\mathrm{Au}$ lieu de se replier dans l'intimité de l'espace privé puis dans celle tout aussi frileuse des romans sentimentaux (écrits et lus par des femmes en majorité et adressés à des lectrices), elles finissent par la dynamiter. Il s'agirait d'indigner le lecteur, de le provoquer, de le violer même dans un coup pour coup d'une grande violence littéraire et médiatique. Car rien ne se passe seulement devant la feuille blanche, il faut aussi rendre des comptes, aller sur les plateaux de télévision et affronter le public, l'opinion. À l'automne 1999, Christine Angot se présente à la télé sur le plateau de Bernard Pivot. Son roman L'Inceste fait scandale parce qu'il remet en question les limites du dicible à tous les niveaux (le viol, l'inceste, l'homosexualité, le mariage...) de la vie et du texte. Puis viendra Pourquoi le Brésil, un roman où elle raconte sa relation avec le journaliste Pierre-Louis Rozynès, remettant à sa place toute la perspective romantique de l'amour (une perspective déjà bien malmenée d'autre part dans d'autres textes).

Christine Angot désigne ses œuvres comme des «romans» indiquant ainsi qu'elle assume le pacte littéraire mais elle bouscule le pacte autobiographique et se fait le porte-parole d'une remise en question déjà revendiquée par d'autres.

Feminismo/s 34, diciembre 2019, pp. 121-141 
La première de la nouvelle vague du TOUT Dire est bien sûr l'incontournable Annie Ernaux. Elle aussi aura le mérite de consigner sans détour tout ce qui concerne la vraie vie de l'intérieur, et cela dès 1984 avec son texte La Place. La fameuse écriture blanche prend toute sa splendeur dans Les Années, un texte où elle parvient à mettre en relation son enfance personnelle sur fond de changements sociétaux, assumant ainsi la double appartenance à un milieu et à une condition.

Virginie Despentes est également un important point de repère si on souhaite mieux connaître la nouvelle autofiction proposée par les écrivaines contemporaines. Elle a contribué à décloisonner le champ d'écriture féministe et propose toute une réflexion très percutante sur les notions d'identités -sexe, genre- et une remise en question du féminisme de la deuxième vague.

Chloé Delaume est la seule à ne pas s'insurger contre le terme d'autofiction. Elle assume qu'elle «est une femme avec personne dedans» dépouillée de tous les clichés, libérée de l'asservissement à l'image et de sa relation au lecteur. «La question du pour qui, du pour qui on écrit, ne s'était jamais posée: je n'écrivais que contre», dit-elle dans Une femme avec personne dedans (130).

Toute la problématique de l'identité, si sensible et si différente chez les femmes et dans leur écriture, est mise en travail chez ces écrivaines et chez d'autres auxquelles nous ferons allusion dans les pages suivantes.

Christine Angot provoque et indigne le lecteur. Elle est considérée par les médias comme la reine de l'autofiction. Sur la quatrième de couverture de Linceste elle assume la situation limite sur laquelle elle se place pour raconter une expérience fondatrice qui marquera toute sa vie: «[...] être juste sur ma limite, m'appuyer dessus comme à la rampe qui monte chez l'avocat. Que tout le monde la voie, ma nullité, mon rien, mon minimum d'être humain, le tout petit écrivain que je suis» (quatrième de couverture).

Le titre du livre et le dévoilement qu'il implique, la formulation ouverte, directe, de l'interdit universel de l'inceste est déjà une provocation en soi. Angot ne contourne pas le délit comme Annie Ernaux avec La Honte, elle n'assume pas la valeur secrète du fait traumatique comme Niki de Saint-Phalle autrice de Mon secret (1994), elle y va directement avec des personnages réels, son ex-mari Claude et son amie Bénédicte et bien sûr de la fiction. Mais le jeu consiste à sortir du «politiquement correct» dans l'intention de nommer 
l'innommable, de mettre en scène les tabous de la société au moyen d'une énonciation sans autocensure.

Christine Angot confirme dans Linceste qu'elle fait le récit vrai d'une expérience réelle qu'elle a vécue durant son adolescence, de 14 à 16 ans, pendant deux années consécutives avec son père. Cette expérience traverse son œuvre et marque tous ses rapports, en particulier sa relation avec sa fille Léonore. Elle utilise l'écriture comme une thérapie et brandit son stylo comme (selon ses propres mots) un «pénis sadique» (152).

Une année plus tôt, dans son essai L'Usage de la vie, elle affirmera: «Les écrivains ne devraient jamais cesser d'écrire leur vie en fait. Avec le doute qui plane. Sur la vérité.» (10).

Elle mêle aussi à l'espace autofictif des définitions tirées du Dictionnaire de la psychanalyse. Cette articulation entre le discours du narrateur et le discours $\mathrm{du}$ dictionnaire (le discours du «savoir»), lui permet de mettre en contact l'universel et le particulier, le singulier, l'exception. Elle donne au lecteur tous les moyens de voir ce qu'il ne veut pas voir, ou qu'il verra en surmontant sa pudeur ou son hypocrisie, en assumant aussi son rôle de voyeur.

Quant à la narratrice de cette histoire «vraie» à l'origine, elle tente de faire émerger sur la page blanche le sujet qu'elle n'a, comme elle le dira, pas choisi: «Écrire n'est pas choisir son récit. Mais plutôt le prendre, dans ses bras» (174).

Ce caractère de révélation n'est pas le propre de cette écrivaine. Cette émergence du moi par l'écriture est présente dans de nombreux textes d'autofiction; Annie Ernaux elle aussi le reconnaît et le commente dans L'écriture comme un couteau.

Pas plus que Nathalie Sarraute je n'aime figurer dans la rubrique «écriture féminine». Il n'y a pas de division de la littérature intitulée «écriture masculine», c'est-à-dire rattachée au sexe biologique ou au genre masculin. Parler d'écriture féminine, c'est de facto faire de la différence sexuelle -et seulement pour les femmes- une détermination majeure à la fois de création et de réception: une littérature de femme pour les femmes.

[...] Cela dit, je suis persuadée qu'on est le produit de son histoire et que celle-ci est présente dans l'écriture. Donc comptent le roman familial, le milieu d'origine, les influences culturelles et bien évidemment la condition liée au sexe. J'ai une histoire de femme... (98-99) 
A côté de la valeur sociologique du discours, il y a aussi et surtout la découverte: Proust précise «La vraie vie, la vie enfin découverte et éclaircie, la seule vie par conséquent réellement vécue, c'est la littérature». (150)

Mais le dévoilement et la révélation de cette découverte intime, cachée, suppose pour beaucoup une «double obscénité»:

Ce qu'on me reproche, c'est une double obscénité, sociale et sexuelle. Sociale, parce que, dans des livres comme La place, Une femme, La honte, mais aussi Journal du dehors, je fais de l'inégalité des conditions, des cultures, la matière du texte, en évitant le populisme qui serait tellement rassurant, acceptable... Sexuelle, parce que dans Passion simple, qui a mis le feu aux poudres, j'ai décrit tranquillement et précisément la passion d'une femme mûre-vécue sur le mode adolescent et celui de la «romance» mais aussi très physique,--sans les marques affectives, la déploration, sans cette «romance» justement qu'on attend dans les écrits des femmes. De plus, une transgression des genres: il s'agit d'un récit autobiographique... (107-108)

Le caractère répétitif est un trait fréquent dans l'écriture de soi par le fait du ressassement permanent d'un même évènement traumatique vu sous diverses perspectives et à différents moments de la vie et de l'écriture. C'est le cas du texte d'Angot intitulé Une semaine en vacances publié en 2012, qui consiste en une re- écriture de l'inceste à la troisième personne. Annie Ernaux, on s'en souvient, procède de la même manière proposant le récit d'une passion et le journal qui reprend cette même histoire (il s'agit du journal Se perdre, 300 pages de notes sans retouche et de Passion simple, récit de sa relation amoureuse).

Comme dans L'Usage de la photo, Angot dans Rendez-vous (2006) choisit de créer un texte qui met en jeu la liaison qu'elle vit avec Éric et qu'elle lira en public. Comme les photos et les textes croisés d'Annie Ernaux et Marc Marie, cette espèce de performance écrite/orale est une interpellation à double voie: Adresse au lecteur, adresse et dialogue envers l'autre, exhibition de l'intimité et du désir. Comme le dira Ernaux dans un entretien lors de la parution de son livre: «Je n'attends pas de la vie qu'elle m'offre des sujets, mais des organisations nouvelles d'écriture. Les photos ont joué ce rôle de déclencheur, de catalyseur même, pour écrire ce qui m'arrivait, notre rencontre, le cancer, tout ce que jusque- là je ne pouvais pas écrire, qu'il me semblait impensable d'écrire» (Paru sur le site Gallimard au moment de la publication du livre).

Feminismo/s 34, diciembre 2019, pp. 121-141 
Limmédiateté de ces actions (rappelons-nous ce que fait dans ce même esprit Hervé Guibert avec la sur-exposition de sa maladie et de son agonie ou Sophie Calle partageant avec son auditoire sa rupture), nous met en contact avec l'étroite relation entre vie et écriture et le besoin d'utiliser le texte comme un élément de plus dans la relation.

En quoi consistera l'engagement dans ce type d'écriture? Comme chez Despentes, comme chez Millet, de nouveau comme chez Ernaux et aussi chez Leduc, Angot provoque le public et l'oblige à prendre parti en le situant face à ce qu'il évite de savoir ou de voir. Elle emboîte le pas à d'autres qui, comme elle, sont allés jusqu'au bout du dire s'insérant dans une «culture de la confession» selon les termes de Robert Dion avec cependant un interdit de plus à surmonter: le fait d'être née femme, ici et maintenant, dans une société où l'impudeur des femmes n'est pas reçue exactement de la même manière que celle des hommes.

Tout ce qui est obscène et tabou et surtout tout ce qui est ambigu, tout ce qui brouille les pistes provoque un débat médiatique et répond au besoin jamais innocent d'indigner (voir l'article de Francesca Forcolin sur ce sujet).

Ce procédé de la provocation est sans aucun doute une manière d'engagement. Au regard des procès et des accusations qui proviennent de l'impact de récits autofictionnels sur l'opinion publique (Marie Darrieussecq accusée par Marie Ndiaye, polémique et accusations entre Camille Laurens et Marie Darrieussecq, condamnation de Christine Angot pour préjudice moral, etc...) on mesure la difficulté à séparer le factuel de la fiction d'une part, et le goût du public pour le déballage qu'il produit.

Le tapage médiatique entretenu entre le public et les auteures est également favorisé par le choix de thèmes comme le sexe, la pornographie ou l'érotisme, surtout s'ils sont traités par des femmes. On constate d'ailleurs qu'elles sont nombreuses à choisir ce domaine: Alina Reyes, Catherine Breillat, Catherine Millet, Françoise Rey, Christine Angot, Virginie Despentes, sont quelques-unes parmi les plus connues.

Le discours amoureux évolue et se caractérise par une distance vis-à-vis de la passion érotique décrite selon le protocole traditionnel. Même quand elle est présente, la passion est analysée avec une lucidité et une liberté qui n'a pas d'équivalent dans la littérature antérieure écrite par des femmes. Le modèle 
féminin change, comme nous le dit Catherine Breillat dans Pornocratie, et les écrivaines proposent un nouveau discours:

Les femmes sont ouvertes à tout vent. C'est cela que les hommes supportent mal [...] Ils ont peur qu'elles ne leur appartiennent pas. Ils ne croient pas en la liberté essentielle. Ils brandissent leurs cadenas, leurs ceintures et leurs préceptes de chasteté, leur morale obtuse car ils ont besoin toujours de se rassurer. Pourtant, ils le savent, il ne faut jamais demander de preuve, car alors, il n'y a pas de validité de l'amour. Il faut croire. Les femmes sont à l'image de Dieu. Croire ce qui est promis sans exiger de le posséder. (28)

Catherine Breillat propose une idéalisation absolue de la position féminine par rapport à l'homme aliéné. Sa mise en scène de la sexualité apparaît comme une dénonciation du machisme, du phallocentrisme et l'assomption d'une nouvelle jouissance amoureuse dont seules les femmes auraient le secret.

Catherine Millet est un autre et brillant exemple du nouveau discours sur la sexualité. Elle entreprend de raconter sa vie sexuelle à visage découvert, dans une langue précise et neutre, afin de produire un témoignage, dans l'intention d'établir une vérité sur sa singularité. Dans son récit, constitué de divers autoportraits à des moments différents de sa vie, les scènes sexuelles se succèdent en nombre considérable sans que jamais la sexualité ne soit en connexion avec le sentiment amoureux.

Elle évoque, comme Angot dans Une semaine en vacances, un fantasme de soumission et de passivité, une attitude vacante qui l'éloigne de la question du désir et des difficultés de la rencontre. Millet adopte une attitude différente de celle de Breillat et met l'accent sur une sexualité qui évite les rapports de séduction. Elle accomplit cette poétique de l'orgie dont parle Anne Garréta dans Pas un jour (152), dans le cadre d'un récit autobiographique soutenu par un titre qui met en relation l'instance fictive et le nom de l'auteure qui surmonte le titre dans un excès de lisibilité et un désir d'extimité ou d'intimité surexposée suivant le titre du livre de Serge Tisseron publié en 2001, alliée à une grande violence de visibilité. C'est aussi dans ce cas la réaffirmation d'un moi social sans voile, sans filtre et une promesse de témoignage singulier. Christine Millet confère à la vie ordinaire un statut symbolique et elle en fait un ready made (dans la mesure où elle expose sur l'autel de l'institution littéraire sa vie privée dans l'intention d'en faire un objet d'art). Lobscénité est une offense et un appel, ou plutôt un rappel des forces en présence dans 
l'intention de bousculer l'ordre établi et ses fondements dualistes: bousculer l'ordre sexué, s'attribuer le droit de faire ce que l'Autre fait en toute impunité s'appuyant sur une légitimité de nature à laquelle le féminin n'a pas systématiquement accès. On constatera que l'œuvre de Millet est fondamentalement dérangeante.

Lindignation et la volonté de bousculer l'ordre établi accompagne et traverse les œuvres de presque toutes les écrivaines contemporaines s'adonnant à l'autofiction. Virginie Despentes est elle aussi un cas extrême qui bouscule les notions ancrées dans la société et dans les textes. Son essai King Kong Théorie, publié en 2006, se présente comme un manifeste pour un nouveau féminisme. Il s'agit d'un récit incorporant les traits de l'autofiction qui a comme objectif de démonter les modèles du patriarcat d'une part et du féministe à l'usage d'autre part. «Tout foutre en l'air» serait la maxime principale de Despentes dès ses débuts (146).

Déjà dans sa fiction Baise-moi en 2000, elle mettait en scène des personnages aux identités socialement reçues comme incohérentes et contraires au schéma établi. Ses personnages Manu et Nadine, parmi d'autres, lui permettent de réviser la définition du féminin et d'ouvrir un débat basé sur le doute identitaire.

Dans King Kong Théorie, elle utilise la narration d'épisodes de sa vie, allant du viol à la prostitution; cette mise en scène très risquée lui permet de poser les jalons politiques d'une nouvelle identité, tout en se faisant le porte-parole de certains groupes bien déterminés: «J'écris de chez les moches, pour les moches, les frigides, les mal-baisées» (9). Elle entreprend aussi une révision critique de sa production antérieure et propose une série de lectures qui appuient sa théorie. Elle déconstruit le stéréotype féminin et aborde des thèmes tabous comme le viol, la prostitution et la pornographie ouvrant le débat sur une polémique très provocante mais cependant motivée. Que cherche-t-elle? Elle tente de faire éclater les codes en se ralliant à un féminisme porno punk tout en partant de l'analyse de son propre cas: «L'envie d'écrire ce livre vient de pas mal d'endroits différents, il vient aussi de l'histoire du film Baise-moi et des interviews auxquels nous avons répondu après sa sortie» (Arbizu et Belin).

Ce texte entre dans la catégorie de ce que Vincent Landry appelle de l'autofiction théorique «un genre à la croisée de l'autofiction et de l'essai», 
en référence à la fiction théorique des écrivaines féministes québécoises des années 70-80. Tout comme dans l'autofiction, les écrivaines de fiction théorique font éclater les normes des genres littéraires, normes fondées sur une tradition patriarcale qui contraint la femme à occuper une position altérisée au sein du champ culturel, et utilisent le langage, l'écriture du soi, la fiction et le discours social pour se libérer d'un régime traditionnel. Ce type de discours incluant plus ou moins ouvertement la théorie dans la fiction a contribué «à développer une mémoire de l'origyne [sic] et à exprimer des valeurs neuves» (Dupré 130).

Lécrivaine croise la fiction et la théorie développant ce que Madeleine Ouelette considère comme de l'énergie profanatrice abordant l'expérience du viol et de la prostitution dans son récit, mais elle a aussi le mérite de secouer l'éternelle passivité et la honte coupable qui accompagne ce type de traumatisme. Contrairement à l'opinion selon laquelle l'écriture du traumatisme est curative, elle nie quant à elle toute relation thérapeutique entre l'expérience et la narration des faits et elle affirme ne pas rechercher la consolation par l'écriture et la confession; en fait, elle n'en cherche aucune, prétendant éliminer tout complexe de culpabilité. Ce qu'elle désire et obtient, c'est la capacité de se poser comme sujet singulier et libre donnant à sa vie une valeur d'intérêt littéraire: «Je n'échangerais ma place contre aucune autre, parce qu'être Virginie Despentes me semble être une affaire plus intéressante à mener que n'importe quelle autre affaire» (9).

Despentes refuse l'asepsie des politiques néolibérales et cherche à revenir à l'activisme en s'instaurant comme une passeuse d'idées pour toutes et pour tous. Elle déconstruit «l'idéal de la femme blanche, séduisante mais pas pute, bien mariée, mais pas effacée, travaillant mais sans trop réussir» (13).

A la différence d'autres écrivaines déjà citées, elle fait du politique sans exclusion. Son discours sur les rapports de domination, sur les questions identitaires concernant le sexe et le genre ne s'enferme pas dans un clan ou dans un ghetto et elle reconnait et invoque l'héritage des travaux de féministes comme Angel Davis, Gail Pheterson, Annie Sprinkle, Simone de Beauvoir ou Virginia Woolf, toutes et chacune représentant des féminismes divers et différents prenant ainsi ses distances de toutes pensées dominantes et sectaires.

Comme pour Delaume qui reprenant et détournant la fameuse phrase de Simone de Beauvoir dira que «On ne naît pas Je, on le devient», pour 
Despentes l'autofiction devient un laboratoire d'écriture et de vie où l'ordre établi est ébranlé dans un mouvement où prime le droit à la différence:

Vouloir être un homme? Je suis mieux que ça. Je m'en fous du pénis. Je m'en fous de la barbe et de la testostérone, j'ai tout ce qu'il me faut en agressivité et en courage. Mais bien sûr que je veux tout, comme un homme, dans un monde d'hommes, je veux défier la loi. Frontalement. Pas de biais, pas en m'excusant. (140)

Cette déclaration rejette l'altérité comme destin et comme système social. Elle remet en question autant les hommes que les femmes et ne voit dans ce système binaire que le prétexte des rapports de pouvoir. (142-143)

La féminité toujours selon Despentes est liée à la victimisation et à la peur. Les femmes sont entraînées dès leur plus jeune âge à accepter la domination: «Il faut que ça reste ouvert, et craintif une femme. Sinon, qu'est-ce qui définirait la masculinité?» (King Kong Théorie 48). Dans cette même ligne, elle invoque Camille Paglia qui considère le viol comme un risque inhérent à la liberté des femmes. Cette position extrêmement provocatrice et dangereuse propose une réflexion sur la situation de la femme propriété de l'homme et sur l'idée de viol et la perception qu'en ont la société et les institutions.

L'expérience de la prostitution est également analysée et décrite comme une autre situation d'ultra féminité dédommagée par la mercantilisation. Cette expérience selon elle cesse d'être le symbole de la déchéance: «La prostitution a été une étape cruciale, dans mon cas, de reconstruction après le viol. Une entreprise de dédommagement, billet après billet, de ce qui m'avait été pris par la brutalité» (72).

Tout le discours sous-jacent révèle bien un refus de toute catégorisation et une résistance acharnée à l'assignation identitaire binaire qu'elle décrit dans le récit de son histoire personnelle et dans ses réflexions théoriques. Comme chez Foucault, c'est à travers une profonde transformation du discours sur la sexualité et sur les rapports identitaires établis que Despentes ouvre la voie à un nouveau féminisme.

Chloé Delaume nous ouvre un autre chemin, tout aussi radical bien que moins violent. Elle adopte le discours psychanalytique et donne à l'inconscient sa présence de personnage.

Chloé Delaume est une romancière performeuse qui dès son troisième livre s'applique cette formule ritualisée («je suis un personnage de fiction»)

Feminismo/s 34, diciembre 2019, pp. 121-141 
pour se mettre en scène. Le nom qu'elle se donne doit beaucoup à la littérature, plus précisément à Boris Vian et à son personnage Chloé du roman L'écume des jours, ainsi qu'à Antonin Artaud auteur de L'Arve et l'Aume'.

Ce double parrainage, sous le signe de la fantaisie et de la folie, accompagne l'auteur dans son labyrinthique parcours qui l'amène à explorer toutes les formes des médias en alliance avec ses textes littéraires dans l'intention de faire de la littérature sa vie. Héritière de l'Oulipo et de Raymond Roussel ce grand magnétiseur des temps modernes, selon une belle formule d'André Breton, elle convertit son œuvre en un grand chantier expérimental faisant de sa vie la matière de son œuvre et aussi de son œuvre le battement de cœur de sa propre vie. Cette double vie entre le factuel et le fictionnel est représentée par deux blogs qui proposent la double version, réel/virtuel. Elle dit dans le premier chapitre de son livre Une femme avec personne dedans: "Je m'appelle Chloé Delaume, je suis un personnage de fiction. Un être d'autofiction. Qui à maintes reprises engage son lecteur à s'écrire par lui-même, à donner à sa vie une forme inédite dont il est le héros » (13) et elle ajoute: "Je m'appelle Chloé Delaume. Je suis un personnage de fiction. Livre et vie s'entremêlent, mon Moi en trois parcelles, auteur, narratrice, héroïne» (17)

Cette mise en scène très étudiée est une fable de soi à partir du vécu mis en fiction. Le moi pour elle et comme pour beaucoup d'autres (souvenons-nous d'Hervé Guibert) n'existe que par la grâce de l'énonciation, mettant en relation l'imposture romanesque et la posture autobiographique plus conventionnelle.

Les moyens dont elle dispose et qu'elle utilise avec ingéniosité sont vastes: le roman interactif Dans ma maison sous terre, pétri d'intertextualité, La nuit je suis Buffy Summers, où l'auteure devient un personnage de jeu numérique embrayeur de fanfiction, ou encore personnage aussi vampirisé par l'entreprise de décervelage qu'implique la télévision dans J'habite la télévision.

La liste est longue et mérite qu'on s'y arrête, mais la question à poser dans notre contexte sera la suivante: dans quelle mesure le mélange des supports et leurs relations permettent de créer une fiction nouvelle et inédite ainsi qu'un brouillage des catégories (biographique, artistique, etc.). Lusage de

1. Chloé procède à son tour d'une chanson arrangée par Duke Ellington intitulé «Song of swamp», la chanson des marais. Chloé personnage de Vian meurt d'un nénuphar dans les poumons.

Feminismo/s 34, diciembre 2019, pp. 121-141 
l'intermédialité chez Delaume et chez de nombreux écrivains contemporains répond au besoin de se libérer des vieux habits de la littérature. Plus encore s'il s'agit de littérature écrite par des femmes qui ne se reconnaissent pas dans le langage patriarcal dont on les affuble dès l'enfance. Dans un article extrêmement évocateur de Silvestra Mariniello, «Cambiar la tabla de operación. El médium intermedial», qui définit l'intermedialité comme un espace hybride où les discours se rencontrent: images, écritures en tous genres, photos, blogs, etc. (et qui montre comment cette discursivité en mouvement perturbe le discours littéraire et met l'accent sur la matérialité des supports, sur le medium ou milieu), l'auteure nous propose l'exemple d'un scénario de film de Pasolini intitulé Le père sauvage et nous montre comment et avec quelle difficulté s'effectue le passage d'une culture à l'Autre et comment le personnage doit puiser dans les images de sa propre culture pour passer d'un monde à l'autre. Cette anecdote que nous transmet Pasolini reprise par Mariniello nous rapproche en partie de l'entreprise de Chloé Delaume dans son besoin de réinventer un langage qui puisse révéler les états successifs de son être. Les techniques audiovisuelles et numériques qu'elle utilise jouent le rôle d'un pont entre les sens et le monde, entre le doute sur la possibilité de la connaissance de soi et les divers moyens technologiques pour donner une image de cette permanente fragmentation qu'elle décrit.

Tout ce débordement de ressources nous reconduit à chaque fois vers Une femme avec personne dedans, mais non «sans personne». Le roman explore ce vide de «l'héroïne», remet en question l'identité figée ainsi que la trop confortable évolution que propose l'auteur du roman traditionnel; Delaume s'insurge contre ce schéma et permet l'ouverture à tous les possibles, pour convertir l'autofiction en un laboratoire du sujet et de ses incertitudes.

Comme le disait déjà en 1996 Marie Darrieussecq dans son article déjà cité «Lautofiction un genre pas sérieux», publié dans la revue Poétique, l'autofiction doit assumer une impossible sincérité ou objectivité et y intégrer une part de brouillage et de fiction due en particulier à l'inconscient. Chloé Delaume entre parfaitement dans ce cadre et lui donne vie en utilisant tous les moyens dont elle peut disposer.

Comme on l'a vu au cours de ces pages, l'espace autofictif créé par les écrivaines est investi de nouvelles caractéristiques de style et d'intention. 
Dans la plupart des cas et avec plus ou moins d'intensité, cette écriture de soi implique une forme de rupture ou de dissidence face aux vieux modèles.

Les filles du Patriarcat (et aussi parfois les fils) n'ont cessé de s'élever contre des représentations vidées de leur sens et les femmes tentent d'adapter le langage à leur image. Face à la relative véracité des faits, les femmes se battent sur tous les fronts du traumatisme -structurels et historiques- selon la terminologie de Dominick La Capra, car dans leur discours la psyché de chaque individu et la mémoire des faits ont été constamment manipulés et soumis au contrôle d'un monde chargé de contraintes sociales et symboliques qui souvent font obstacle à la création telle que la conçoivent ces écrivaines contemporaines; Plus que d'un brouillage on devrait parler d'un véritable chambardement qui secoue les réalités et les énonciations, fracturant l'échafaudage littéraire et la répartition des rôles. Dans le cadre de l'écriture autofictionnelle produite par des femmes qui écrivent dans une langue qui est censée appartenir à tous, le thème de la sexualité est apparu comme un phénomène qui a déjà une longue trajectoire mais qui a fait l'objet d'un déni d'antérioritée .

Avant l'époque que nous avons cernée, à partir du début du XXe siècle, il existe comme nous l'avons dit des exemples marquants et singuliers comme celui de Violette Leduc auteure du fameux roman préfacé par Simone de Beauvoir Thérèse et Isabelle, écrit en 1954, publié dans une version censurée en 1964 et finalement édité dans sa version intégrale en 2000; Marguerite Duras, auteure de l'Amant, Annie Ernaux, toujours présente et combattive, toutes trois rejointes par de nouvelles figures qui ont en partage la violence de l'expression et l'utilisation de la sexualité et du désir comme instrument de la construction identitaire du sujet féminin dans la perspective des femmes. Le choix du récit d'autofiction facilite le témoignage d'une expérience personnelle (même si elle est constamment fantasmée) dans l'intention d'accéder à ce que Michel Bozon définit comme une «politisation de l'intimité» qui place dans la sphère publique un débat sur la sexualité mise en lien avec la question de la construction de l'identité. Cette question du désir et du sexe est aussi le

2. C'est-à-dire d'un refus à inscrire dans une histoire et une tradition littéraire l'écriture féminine, qui plus est sur la sexualité, ce qui fait de chaque nouvelle prise de parole une nouveauté.

Feminismo/s 34, diciembre 2019, pp. 121-141 
point de départ d'autres questions de première importance qui prennent place dans cette littérature autofictionnelle: Les relations dans toute leur diversité, le pouvoir, la création, pour ne citer que les plus importantes.

Michel Foucault avait déjà dans son Histoire de la sexualité envisagé cette histoire du point de vue de la répression en vue du contrôle de la société néo libérale. La reprise du thème aujourd'hui avec cette «mise hors de soi» du désir dans la perspective du féminin est dans une certaine mesure une reprise de cette approche. Lhistoire de la soumission et de la victimisation du corps féminin est réécrite sous l'angle du sujet écrivant. Même si à certains moments le personnage adopte le rôle passif que l'autre lui assigne, il n'en demeure pas moins comme un témoin narrateur actif et explicite qui raconte comment il a été soumis, et jusqu'où. La double écriture d'Annie Ernaux, son journal et son récit sont là pour témoigner des contradictions et reprendre en mains son délire. Les divers supports utilisés par Chloé Delaume lui permettent aussi de chercher des langages qui puissent exprimer jusqu'au bout qui elle est ou qui elle n'est pas et ce qu'elle donne à voir.

De même, dans Une semaine en vacances, Christine Angot se place au centre de la représentation et donne à voir un objet sexuel doublé d'un sujet narratif qui contrôle (bien qu'à posteriori) la scène de soumission. Cette attitude très risquée est également partagée en d'autres termes par Virginie Despentes ou par Catherine Millet.

Cette forme d'écriture, très provocatrice, très exhibitionniste peut être prise comme un piège qui associerait de nouveau les femmes à l'antique maxime tota mulier in utero ou au contraire comme un empowerment ou une agentivité qui donnerait la possibilité au sujet de créer son identité de genre par la performance, autrement dit par un rituel qui se rejoue constamment, à l'intérieur d'un même texte ou d'un texte à l'autre, tenant compte du fait que l'acte performatif acquière son autorité grâce à la répétition. Cette mise en scène d'un sujet représenté autrement donne la possibilité de changer ou de modifier les perspectives. On pourrait encore pourtant se demander si le changement et la subversion proviennent essentiellement du texte ou si le contexte et les manifestations médiatiques pèsent davantage dans la majeure partie des cas.

Un autre exemple intéressant que nous avons rencontré dans les œuvres citées est celui du thème récurrent de la prostitution: Despentes et Arcan 
exercent la prostitution pendant une période et elles décrivent leur expérience dans une autoreprésentation très variable dans les deux cas. Cependant et en dépit des différences, l'exploration de la situation et le discours sur cette expérience devient potentiellement un instrument politique, une mise à mort du mythe patriarcal du mâle dominant. La relecture de la pornographie est une autre tentative qui va dans la même direction: le scénario de Despentes issu de son roman Baise-moi ou les manipulations des scènes de films pornographiques par Annie Sprinkle répondent, elles aussi, à une volonté de rediriger les perspectives établies selon un rituel jusqu'à présent inamovible. L'essai King Kong Théorie, auquel nous faisions allusion antérieurement, est également au cœur de cette recherche avec l'intention de dénoncer la vulnérabilité dans laquelle les femmes sont enfermées et de retourner la situation d'agression en cessant de les considérer comme le «sexe faible».

Les clichés, les stéréotypes, les mythologies qui peuplent l'imaginaire contemporain, sont revisités par l'écriture des femmes. L'autofiction, l'espace inter-fictionnel tel qu'il est investi par les femmes permet d'exhiber le moi fantasmé et en permanente réinvention, d'interpeler le lecteur, d'entreprendre un dévoilement ambigu entre l'imaginaire et les faits. Linterrogation du «qui suis-je» est étroitement mêlée à une réflexion forte sur le pouvoir de l'écriture; le sujet, le lecteur, est interpelé (ce que Judith Butler appelle précisément la scène d'interpellation). Lidentité sociale souvent incertaine est doublée d'une identité narrative problématisée. Beaucoup d'aspects du sujet sont battus en brèche et posés selon de nouvelles prémisses. C'est donc une histoire du sujet et de tous les sujets qui s'écrit dans les textes que nous venons d'évoquer. Angot, Millet, Delaume, Despentes comme déjà le faisaient Leduc, Duras, Ernaux, esquissent une histoire du sujet au travers de leur propre expérience et de celle de la société qui les a vues naître. Certaines le feront dans un axe plus sociologique, d'autres se submergent dans le grand fleuve de la psychanalyse, d'autres s'attèlent à un engagement féministe provocateur, toutes appellent à la reconnaissance de leur valeur et de leur entreprise, se mettant au centre de l'action et au centre du débat. L'histoire identitaire est loin d'être claire et résolue mais elle avance et l'opinion publique s'en empare. Comme l'a été Rousseau à son époque, interpelé et interpelant, comme l'a aussi été George Sand, tout aussi audacieuse par sa volonté de vivre et d'écrire sous le regard de la société qui la juge, représentée comme une reine par

Feminismo/s 34, diciembre 2019, pp. 121-141 
Nadar le grand photographe de l'époque, nos écrivaines assument leur double challenge, celui de la littérature comme recherche, comme éclatement de la fiction ancienne comme dira Ernaux dans l'Écriture comme un couteau, mais aussi l'écriture comme recherche du sujet quand on est une femme qui écrit même si, comme on l'a vu le sexe et le genre sont vécus et revisités selon des procédés et des intentions différents.

\section{BIBLIOGRAPHIE}

Angot, Christine. L'Inceste. Paris: Stock, 1999.

Angot, Christine. L'usage de la vie. Edition Mille et une nuits, 1999.

Angot, Christine. Pourquoi le Brésil. Paris: Stock, 2002.

Angot, Christine. Le marché des amants. Paris: Points, 2008.

Angot, Christine. Rendez-vous. Paris: Gallimard, 2008.

Angot, Christine. Une semaine de vacances. Paris: Flammarion, 2012.

Arbizu, Susana et Henri Belin. «King Kong théorie: entretien avec Virginie Despentes».

Mauvaiseherb'sweblog, 11.09.2008. <http://mauvaiseherbe.wordpress. com/2008/09/11/king-kong-theorie-entretien-avec-virginie-despentes-/>

Bereni, Laura et Trachman, Mathieu. Le genre, théories et controverses. Paris: PUF, 2014.

Blanckman, Bruno. Les récits indécidables: Jean Echenoz, Hervé Guibert, Pascal Quignard. Villeneuve d'Ascq: Presses universitaires du Septentrion, coll. «Perspectives», 2000.

Bourdieu, Pierre. «La marchandisation de la culture». Inter: art actuel 80 (20012002): 5-9.

Bozon, Michel. «Les cadres sociaux de la sexualité». Sociétés contemporaines 41-42 (2001): 5-9.

Breillat, Catherine. Pornocratie. Paris: Denoël, 2004.

Darrieussecq, Marie. «Lautofiction, un genre pas sérieux». Poétique 107 (1996): 369-380.

Debord, Guy. La société du spectacle. Paris: Buchet/Chatel, 1967.

Delaume, Chloé. La nuit je suis Buffy Summers. Editions ERE, 2007.

Delaume, Chloé. Dans ma maison sous terre. Paris: Seuil, 2009.

Delaume, Chloé. Une femme avec personne dedans. Paris: Le Seuil, 2012.

Despentes, Virginie. Baise-moi. Paris: Flammarion, 1999.

Despentes, Virginie. King Kong Théorie. Paris: Grasset, coll. «Livre de poche», 2006. 
Dion, Robert et al. «Vie en récit. Formes littéraires et médiatiques de la biographie et de l'autobiographie». Questions de communication 14 (2008): 343-245.

Doubrovsky, Serge. Fils. Paris: Éditions Galilée, 1977.

Dupré, Louise. «Quatre esquisses pour une morphologie», La théorie un dimanche, Montréal:1988.

Ernaux, Annie. La Place. Paris: Gallimard, 1984.

Ernaux, Annie. Une femme. Paris: Gallimard, 1988.

Ernaux, Annie. Passion simple. Paris: Gallimard, 1992.

Ernaux, Annie. Journal du dehors. Paris: Gallimard, 1993.

Ernaux, Annie. Se perdre. Paris: Gallimard, 2001.

Ernaux, Annie. Lécriture comme un couteau. Paris: Stock, 2003.

Ernaux, Annie. L'usage de la photo. Paris: Gallimard, 2005.

Ernaux, Annie. Les années. Paris: Gallimard, 2008.

Forcolin, Francesca. «Catherine Angot, le désir d'indigner le lecteur; la société violée par l'(auto) fiction». Carnets [mis en ligne le 19 juin 2018], Première série-3 Numéro spécial (2011): 51-61.

Foucault, Michel. Histoire de la sexualité. Paris: Gallimard, 1976/1984.

Havercroft, Barbara. «Espace autofictif, sexuation et deuil chez Denise Desautels et Paul Chanel Malenfant». Sexuation, espace, écriture. La littérature québécoise en transformation. Eds. Dupré, Louise, Lintvelt, Jaap, Paterson, Janet M. Québec: Nota bene, 2002.

Landry, Vincent. «Virginie Despentes et l'autofiction théorique: étude de King Kong Théorie». Revue politiqueer, 29 décembre 2012.

Lash, Christopher. La culture du narcissisme. Éditions Climats, 1979.

Laurens, Camille. Ni toi ni moi. Paris: Gallimard, 2008.

Leduc, Violette. Thérèse et Isabelle. Paris: Gallimard, 2000.

Leibovici, Solange. «Autoreprésentation et stade du miroir: quelques remarques sur les liens entre l'autobiographie et la psychanalyse ». Gradiva (2003): 40-45.

Lipovesky, Gilles. L'ère du vide. Essais sur l'individualisme contemporain. Paris: Gallimard, 1983.

Mariniello, Silvestra. «Cambiar la tabla de operación. El médium intermedial». Acta Poetica 30-2 (automne 2009): 59-85.

Schmitt, Arnaud. «La perspective de l'autofiction». Poétique 149 (2007): 15-29.

Tisseron, Serge. Lintimité surexposée. Paris: Hachette Littérature, 2001. 